\title{
Preparation of Layer-by-Layer Films Composed of Polysaccharides and Poly(Amidoamine) Dendrimer Bearing Phenylboronic Acid and Their pH- and Sugar-Dependent Stability
}

\author{
Kentaro Yoshida ${ }^{1}$, Keisuke Suwa ${ }^{2}$ and Jun-ichi Anzai ${ }^{2, *}$ \\ 1 School of Pharmaceutical Sciences, Ohu University, 31-1 Misumido, Tomita-machi, Koriyama, \\ Fukushima 963-8611, Japan; k-yoshida@pha.ohu-u.ac.jp \\ 2 Graduate School of Pharmaceutical Sciences, Tohoku University, Aramaki, Aoba-ku, Sendai 980-8578, Japan; \\ b4ym1017@s.tohoku.ac.jp \\ * Correspondence: junanzai@mail.pharm.tohoku.ac.jp; Tel.: +81-22-795-6841 \\ Academic Editor: Andrew J. Ruys \\ Received: 21 April 2016; Accepted: 25 May 2016; Published: 28 May 2016
}

\begin{abstract}
Layer-by-layer films composed of polysaccharides and poly(amidoamine) dendrimer bearing phenylboronic acid (PBA-PAMAM) were prepared to study the deposition behavior of the films and their stability in buffer solutions and in sugar solutions. Alginic acid (AGA) and carboxymethylcellulose (CMC) were employed as counter-polymers in constructing LbL films. AGA/PBA-PAMAM films were successfully prepared at $\mathrm{pH}$ 6.0-9.0, whereas the preparation of CMC/PBA-PAMAM film was unsuccessful at $\mathrm{pH} 8.0$ and 9.0. The results show that the LbL films formed mainly through electrostatic affinity between PBA-PAMAM and polysaccharides, while, for AGA/PBA-PAMAM films, the participation of boronate ester bonds in the films was suggested. AGA/PBA-PAMAM films were stable in the solutions of $\mathrm{pH}$ 6.0-9.0. In contrast, CMC/PBA-PAMAM films decomposed at pH 7.5-9.0. The AGA/PBA-PAMAM films decomposed in response to 5-30 mM fructose at $\mathrm{pH} 7.5$, while the films were stable in glucose solutions. Thus, AGA is useful as a counter-polymer for constructing PBA-PAMAM films that are stable at physiological $\mathrm{pH}$ and decompose in response to fructose.
\end{abstract}

Keywords: layer-by-layer film; pH-sensitive; sugar-sensitive; dendrimer; phenylboronic acid; polysaccharide; alginic acid; carboxymethylcellulose

\section{Introduction}

Layer-by-layer (LbL) films have attracted much attention because of their potential use in separation and purification [1], sensors [2,3], drug delivery [4,5], and so forth. LbL films are prepared by an alternate deposition of synthetic polymers [6-8] and biopolymers including proteins [9-11], polysaccharides [12-14], and DNA [15] on the surfaces of solid substrates and nanoparticles. Synthetic dyes and nanomaterials have also been employed as components of LbL films $[16,17]$.

We have recently prepared stimuli-sensitive LbL films that decompose in response to sugars using phenylboronic acid (PBA)-bearing poly(amidoamine) dendrimers (PBA-PAMAM, Figure 1) for the applications of controlled release [18-20]. The LbL films were constructed by the alternate deposition of PBA-PAMAM and poly(vinyl alcohol) (PVA) $[18,19]$ or glycoprotein (i.e., glucose oxidase, GOx) [20]. It is known that PBA binds diol compounds to form cyclic boronate ester bonds in aqueous media (Figure 2) [21]. Thus, PVA/PBA-PAMAM and GOx/PBA-PAMAM films were stabilized through boronate ester bonds between PBA-PAMAM and diol units in PVA or saccharide chains in GOx, respectively. In fact, PVA/PBA-PAMAM films were fairly stable in the solutions of basic pHs, 
whereas the films decomposed at weakly acidic $\mathrm{pH}$ [19]. On the other hand, GOx/PBA-PAMAM films decomposed to some extent, even at $\mathrm{pH}$ 7.0-8.0, while the films were stable in the solutions with $\mathrm{pH}$ higher than 8.5 [20]. These results show that $\mathrm{pH}$ stability of PBA-PAMAM-containing LbL films depends on the type of counter-polymers used. Other papers also reported $\mathrm{pH}$-dependent stability of LbL films cross-linked with boronate ester bonds [18,22-24]. From the viewpoint of biomedical applications of LbL films, it is a prerequisite for the films to be stable under physiological conditions.

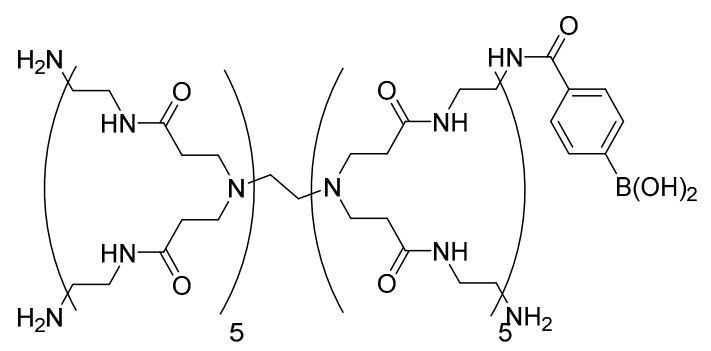

Figure 1. Chemical structure of PBA-PAMAM.

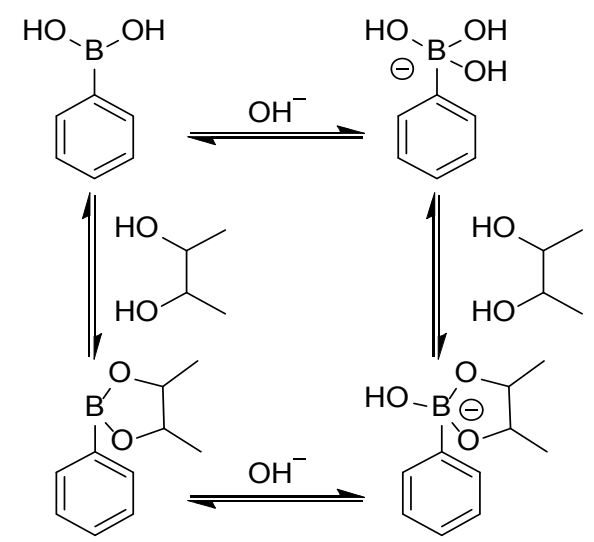

Figure 2. Binding equilibria of PBA to sugar and $\mathrm{OH}^{-}$ion.

In this work, we used anionic polysaccharides, alginic acid (AGA) and carboxymethylcellulose (CMC) as counter-polymers for constructing AGA/PBA-PAMAM and CMC/PBA-PAMAM films to improve the $\mathrm{pH}$ stability as well as sugar response of the films. The LbL films would be stabilized through electrostatic bonds between positively charged PBA-PAMAM and the anionic polysaccharides. In addition, polysaccharides may form boronate ester linkages with PBA-PAMAM in the films because monomer units of the polysaccharides contain 1,2-diol residues. In fact, we have found that AGA/PBA-PAMAM films are stable at a physiological $\mathrm{pH}$, though CMC/PBA-PAMAM films are unstable at $\mathrm{pH} 7.5$ or higher. The present paper reports the preparation of LbL films composed of PBA-PAMAM and AGA or CMC and their $\mathrm{pH}$ stability as well as sugar response.

\section{Materials and Methods}

\subsection{Materials}

PAMAM dendrimer (4th generation, ethylenediamine core, 10\% methanol solution) was purchased from Sigma-Aldrich Co. (St. Louis, MO, USA). AGA and CMC were obtained from Funakoshi Co. (Tokyo, Japan) and Tokyo Kasei Co. (Tokyo, Japan), respectively, and used without further purification. 4-Carboxyphenylboronic acid was obtained from Tokyo Kasei Co. 1-Ethyl-3-(3dimethylaminopropyl)carbodiimide hydrochloride (EDC) and $N$-hydroxysuccineimide (NHS) were obtained from Nacalai Tesque Co. (Kyoto, Japan). 


\subsection{Preparation of PBA-PAMAM}

PBA-PAMAM was prepared as reportedly $[18,19]$. Briefly, NHS ester of 4-carboxyphenylboronic acid was prepared by coupling NHS and 4-carboxyphenylboronic acid in $N, N$-dimethylformamide (Wako Pure Chemicals Industries, Ltd., Osaka, Japan) in the presence of EDC. The reaction mixture was stirred for $1 \mathrm{~h}$ at $0{ }^{\circ} \mathrm{C}$ and then for $12 \mathrm{~h}$ at room temperature. The crude product was purified by silica gel column chromatography (chloroform/methanol/acetic acid (Wako Pure Chemicals Industries, Ltd., Osaka, Japan), 100:10:1). The NHS ester was reacted with PAMAM in water for $12 \mathrm{~h}$ at room temperature. PBA-PAMAM was purified by dialysis in water using Spectra/Por ${ }^{\circledR} 6$ dialysis membrane (molecular weight cut-off: 3500, Spectrum Lab. Inc., Rancho Dominguez, CA, USA). Thus, PBA-PAMAM samples in which PBA residues were attached to $19 \mathrm{~mol} \%$ and $39 \mathrm{~mol} \%$ of the primary amino groups (referred to hereafter as 19\%PBA-PAMAM and 39\%PBA-PAMAM, respectively), as determined by the UV absorption spectra, were obtained.

\subsection{Preparation of LbL Films}

LbL films were prepared on the surface of a quartz slide $\left(50 \times 9 \times 1 \mathrm{~mm}^{3}\right)$ (Yazawa Co., Sendai, Japan), which had been cleaned in a mixture of sulfuric and chromic acids (Wako Pure Chemicals Industries, Ltd., Osaka, Japan). The quartz slide was alternately immersed in a $0.1 \mathrm{mg} \cdot \mathrm{mL}^{-1} \mathrm{AGA}$ or CMC solution and a $0.1 \mathrm{mg} \cdot \mathrm{mL}^{-1}$ PBA-PAMAM solution for $20 \mathrm{~min}$ to deposit AGA/PBA-PAMAM or CMC/PBA-PAMAM films on the surface of the quartz slide. The PBA-PAMAM and polysaccharides solutions were prepared using $10 \mathrm{mM} \mathrm{N}$-cyclohexyl-2-aminoethanesulfonate (CHES) buffer at $\mathrm{pH}$ 9.0, $10 \mathrm{mM}$ 1-[4-(2-hydroxyethyl)-1-piperadinyl]ethanesulfonic acid] (HEPES) buffer at $\mathrm{pH}$ 7.0-8.0, and $10 \mathrm{mM}$ 2-(N-morpholino)ethanesulfonic acid (MES) buffer at pH 6.0. All buffer solutions (Nacalai Co., Kyoto, Japan) contained $150 \mathrm{mM} \mathrm{NaCl}$ (Wako Pure Chemicals Industries, Ltd., Osaka, Japan). The quartz slide was rinsed in the working buffer for $5 \mathrm{~min}$ twice after each deposition. UV absorption spectra of the film-coated quartz slide were recorded in the working buffer on a UV-visible absorption spectroscope (UV-3100PC, Shimadzu, Kyoto, Japan) after each deposition of PBA-PAMAM and AGA or CMC.

\section{4. $p H$-Dependent Stability}

LbL films were deposited on one side of the quartz slide at $\mathrm{pH}$ 6.0. The slide was placed in a quartz cuvette (optical path length, $10 \mathrm{~mm}$ ) filled with a buffer solution. The slide was placed near the sidewall of the cuvette, parallel to the light path, in order to avoid blocking the incident light. The absorbance of the buffer solution was monitored at $242 \mathrm{~nm}$ with gentle stirring to estimate the extent of film decomposition at different $\mathrm{pHs}$. This protocol enables us to record absorption intensity of the buffer solution, not that of the films, in which decomposed film components are dissolved.

\subsection{Sugar-Induced Decomposition}

The sugar-induced decomposition of the films were studied by monitoring the absorbance of $10 \mathrm{mM}$ HEPES buffer solution at $\mathrm{pH}$ 7.5, in which LbL film-coated quartz slide was immersed, upon successive addition of 1-30 mM glucose and fructose (Wako Pure Chemicals Industries, Ltd., Osaka, Japan). All measurements were carried out at room temperature $\left(c a .23^{\circ} \mathrm{C}\right)$.

\section{Results and Discussion}

\subsection{Preparation of LbL Film}

PBA-PAMAM may be built into LbL films because positively charged primary amino groups of PBA-PAMAM are available for the electrostatic binding to carboxylate residues of AGA and CMC. Figure 3 shows UV absorption spectra of (AGA/39\%PBA-PAMAM) $)_{n}$ films $(n=1-10)$ prepared at $\mathrm{pH}$ 6.0, 7.0, 8.0 and 9.0. The absorption band of the films around $240 \mathrm{~nm}$, which originates from 
39\%PBA-PAMAM, increased with increasing the number of AGA/39\%PBA-PAMAM bilayers, showing that (AGA/39\%PBA-PAMAM) ${ }_{10}$ LbL films were successfully constructed at $\mathrm{pH}$ 6.0-9.0. The intensities of the absorption bands of the films prepared at $\mathrm{pH} 6.0$ and 7.0 were higher than those of the films deposited at $\mathrm{pH} 8.0$ and 9.0, suggesting that electrostatic affinity between AGA and 39\%PBA-PAMAM is a main driving force for the film formation. It is envisaged that the density of the positive charges in $39 \%$ PBA-PAMAM, which originates from the primary and tertiary amino groups, is $\mathrm{pH}$-dependent in the range of $\mathrm{pH}$ 6.0-9.0. The $\mathrm{p} K_{\mathrm{a}}$ values of primary and tertiary amino groups of PAMAM are reported to be 9.23 and 6.30, respectively [25]. Thus, the surface of 39\%PBA-PAMAM should be highly protonated at $\mathrm{pH} 6.0$ and 7.0, resulting in strong binding to AGA ( $\mathrm{p} K_{\mathrm{a}}, 3.5-5.0$ [26]) in the film. In contrast, the amount of positive charges is lower at $\mathrm{pH} 8.0$ and 9.0, which limits electrostatic binding of 39\%PBA-PAMAM to AGA. It is also plausible that the LbL films are stabilized in part by boronate ester bonds between 39\%PBA-PAMAM and AGA. A possible participation of boronate ester bonds in the LbL films will be further discussed in the later section in relation to the preparation of CMC/39\%PBA-PAMAM films and their $\mathrm{pH}$ stability.
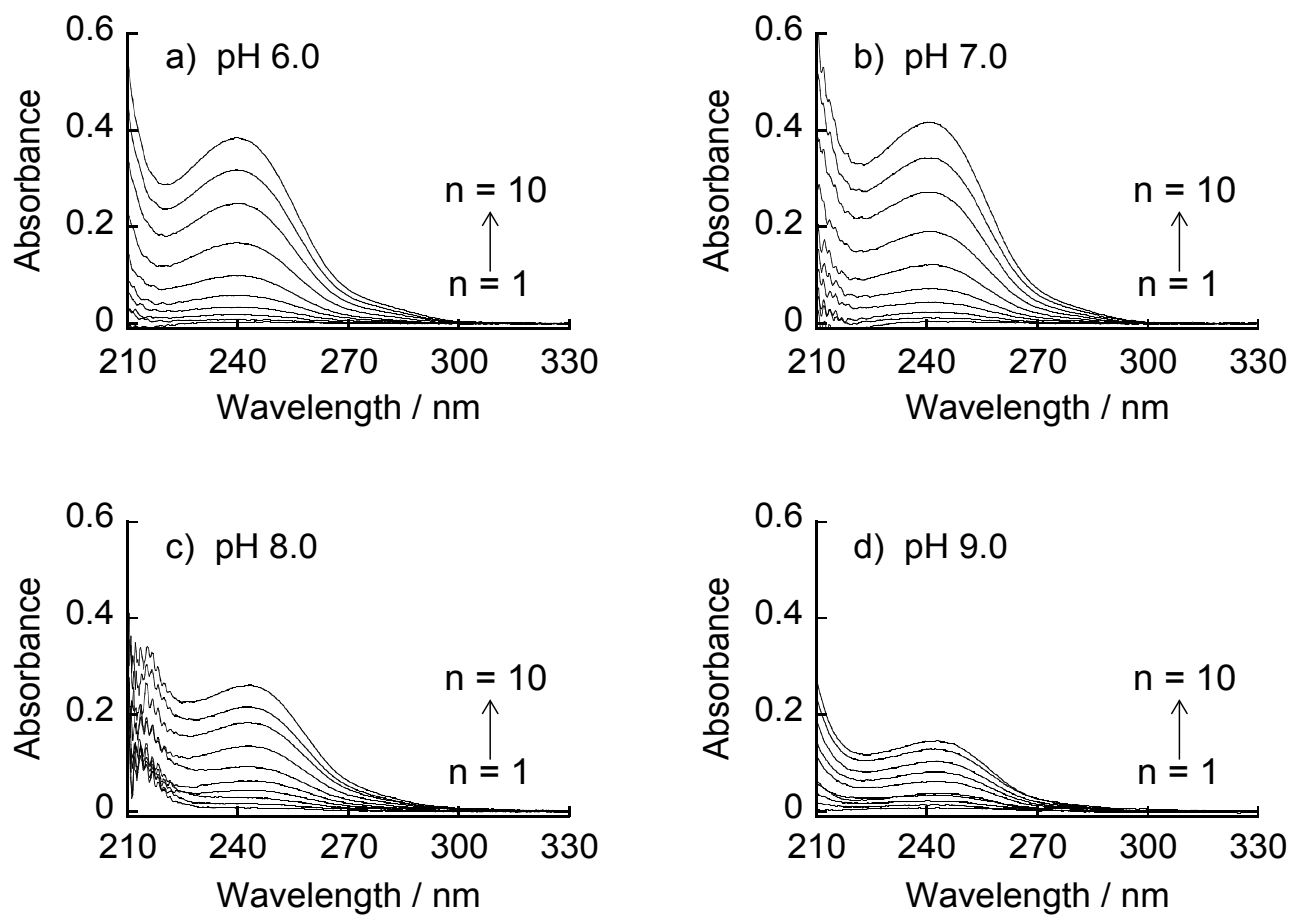

Figure 3. UV absorption spectra of (AGA/39\%PBA-PAMAM $)_{n}$ LbL films $(n=1-10)$ prepared at pH 6.0-9.0. (a) UV absorption spectra recorded at $\mathrm{pH}$ 6.0; (b) UV absorption spectra recorded at $\mathrm{pH}$ 7.0; (c) UV absorption spectra recorded at $\mathrm{pH} 8.0$; (d) UV absorption spectra recorded at $\mathrm{pH} 9.0$.

Figure 4 shows UV absorption spectra of $(\mathrm{CMC} / 39 \% \text { PBA-PAMAM })_{\mathrm{n}}$ films $(n=1-10)$ prepared at $\mathrm{pH}$ 6.0-9.0. The absorption bands of the films increased with increasing the number of depositions at $\mathrm{pH} 6.0$ and 7.0, while film deposition was unsuccessful at $\mathrm{pH} 8.0$ and 9.0. The results suggest that the deposition of (CMC/39\%PBA-PAMAM $)_{10}$ films relies on the electrostatic force of attraction, as in the case for the (AGA/39\%PBA-PAMAM) $)_{10}$ film. The deposition behavior of the $(\mathrm{CMC} / 39 \% \text { PBA-PAMAM })_{10}$ films is somewhat different from that of the (AGA/39\%PBA-PAMAM $)_{10}$ films. First, deposition of the (CMC/39\%PBA-PAMAM $)_{10}$ film was suppressed almost completely at $\mathrm{pH} 8.0$ and 9.0, in contrast to the successful deposition of the (AGA/39\%PBA-PAMAM) ${ }_{10}$ films even at the higher pHs. In addition, the intensities of the $240 \mathrm{~nm}$ band of the (CMC/39\%PBA-PAMAM $)_{10}$ films are always lower than those of the (AGA/39\%PBA-PAMAM) $)_{10}$ films prepared at the same $\mathrm{pH}$. Thus, a larger amount of $39 \%$ PBA-PAMAM was deposited in the (AGA/39\%PBA-PAMAM) 10 films 
than in the CMC-based films. These results cannot be fully rationalized only from the electrostatic binding between 39\%PBA-PAMAM and the polysaccharides because the charge density (or the number of carboxylate residues) of AGA and CMC is nearly comparable. Therefore, the results suggest the participation of a secondary binding force, i.e., boronate ester bonds, in the (AGA/39\%PBA-PAMAM) 10 films. AGA is a polysaccharide composed of D-mannuronic acid and L-gluronic acid, which are derived from D-mannose and L-glucose, respectively, while CMC consists of carboxymethylated D-glucose units. It has been reported that mannose polymer, i.e., mannan, forms boronate ester bonds with PBA polymer to provide LbL films [27]. The binding constant of PBA to D-mannose $\left(13 \mathrm{M}^{-1}\right.$ at $\mathrm{pH} 7.4)$ is higher than that to D-glucose $\left(4.6 \mathrm{M}^{-1}\right.$ at $\left.\mathrm{pH} 7.4\right)$ [21]. These data support that the (AGA/39\%PBA-PAMAM) ${ }_{10}$ films were stabilized in part by boronate ester bonds, which was not the case for the $(\mathrm{CMC} / 39 \% \text { PBA-PAMAM })_{10}$ films.
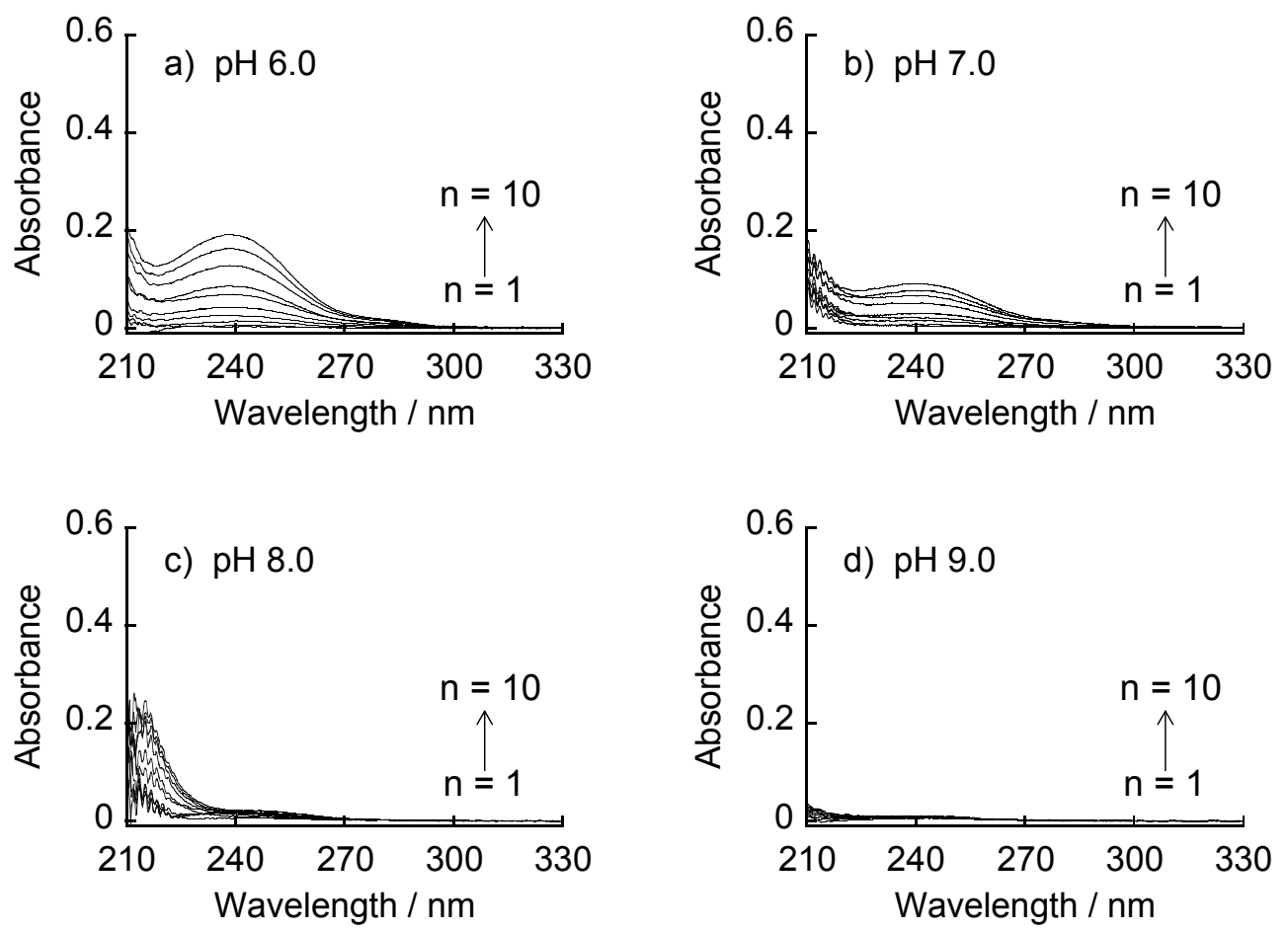

Figure 4. UV absorption spectra of (CMC/39\%PBA-PAMAM $)_{\mathrm{n}}$ LbL films $(n=1-10)$ prepared at pH 6.0-9.0. (a) UV absorption spectra recorded at $\mathrm{pH}$ 6.0; (b) UV absorption spectra recorded at $\mathrm{pH}$ 7.0; (c) UV absorption spectra recorded at $\mathrm{pH} 8.0$; (d) UV absorption spectra recorded at $\mathrm{pH} 9.0$.

In order to evaluate the effect of PBA content in PBA-PAMAM on the film formation, 19\%PBA-PAMAM was used to construct LbL films. Figures S1 and S2 in Supplementary Materials show UV absorption spectra of (AGA/19\%PBA-PAMAM $)_{n}$ and (CMC/19\%PBA-PAMAM $)_{n}$ films $(n=1-10)$ prepared at $\mathrm{pH}$ 6.0-9.0. The deposition behavior of the films was similar to that of the 39\%PBA-PAMAM films. However, absorption intensities in the UV spectra of the $(\mathrm{AGA} / 19 \% \mathrm{PBA}-\mathrm{PAMAM})_{\mathrm{n}}$ and $(\mathrm{CMC} / 19 \% \mathrm{PBA}-\mathrm{PAMAM})_{\mathrm{n}}$ films were always lower than those of 39\%PBA-PAMAM films prepared at the same $\mathrm{pH}$. This is reasonable because the absorption bands around $240 \mathrm{~nm}$ are arising from PBA residues in PBA-PAMAM. The loadings of PBA-PAMAM in the $(\mathrm{AGA} / 19 \% \mathrm{PBA}-\mathrm{PAMAM})_{10}$ and (AGA/39\%PBA-PAMAM) 10 films prepared at $\mathrm{pH} 6.0$ are calculated to be $1.47 \times 10^{-9}$ and $1.23 \times 10^{-9} \mathrm{~mole} / \mathrm{cm}^{2}$, respectively, showing that both the films contain comparable amounts of PBA-PAMAM. The loadings of PBA-PAMAM in the $(\mathrm{CMC} / 19 \% \mathrm{PBA}-\mathrm{PAMAM})_{10}$ and $(\mathrm{CMC} / 39 \% \mathrm{PBA}-\mathrm{PAMAM})_{10}$ films were prepared at $\mathrm{pH} 6.0$ are $9.69 \times 10^{-10}$ and $6.06 \times 10^{-10} \mathrm{~mole} / \mathrm{cm}^{2}$, respectively. Thus, the amount of PBA-PAMAM in the films did not significantly depend on the degree of PBA substitution in PBA-PAMAMs. 


\section{2. $p H$-Dependent Stability}

It is reasonable to assume that PBA-PAMAM films are sensitive to $\mathrm{pH}$ because the amounts of positive charges of the PBA-PAMAM used are dependent on the $\mathrm{pH}$ of the media. The addition of an $\mathrm{OH}^{-}$ion to the boron atom of the PBA moiety results in a tetragonal form, which has a stronger binding affinity than that of the parent trigonal form (Figure 2) [21]. In fact, we have found that PBA-PAMAM films decomposed in response to $\mathrm{pH}$ changes.

Figure 5 shows the decomposition kinetics of the (AGA/39\%PBA-PAMAM) ${ }_{10}$ and (CMC/ $39 \%$ PBA-PAMAM $)_{10}$ films in a buffer solution of $\mathrm{pH} 9.0$. The (CMC/39\%PBA-PAMAM $)_{10}$ film decomposed in the basic solution, whereas the (AGA/39\%PBA-PAMAM) ${ }_{10}$ film did not decompose at all. This shows that the $\mathrm{pH}$ stability of PBA-PAMAM films significantly depends on the type of polysaccharide used. In a separate experiment, the $\mathrm{pH}$ stability of the films was evaluated by immersing the films in solutions of $\mathrm{pH}$ 6.0-9.0 (Figure 6). The (AGA/19\%PBA-PAMAM) 10 and (AGA/39\%PBA-PAMAM) ${ }_{10}$ films were highly stable in the solutions of $\mathrm{pH} 6.0-9.0$, while the $(\mathrm{CMC} / 19 \% \mathrm{PBA}-\mathrm{PAMAM})_{10}$ and (CMC/39\%PBA-PAMAM $)_{10}$ films decomposed at $\mathrm{pH}$ 7.5-9.0. Both the films are electrostatically stabilized at $\mathrm{pH}$ 6.0-7.0 because the dendrimers contain higher amounts of positive charges. However, the amount of positive charges decreased at the higher $\mathrm{pH}$, resulting in the loss of electrostatic affinity of the PBA-PAMAM to CMC. On the other hand, the AGA-based films were stable even at the higher $\mathrm{pH}$ owing to the boronate ester bonds formed between PBA-PAMAM and AGA.

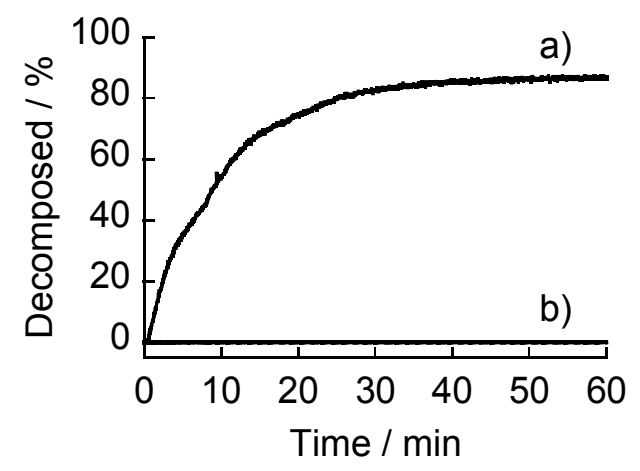

Figure 5. The kinetics of the decomposition of (CMC/39\%PBA-PAMAM) $)_{10}(\mathbf{a})$ and (AGA/39\%PBAPAMAM $)_{10}$ films (b) in buffer solution at $\mathrm{pH} 9.0$.

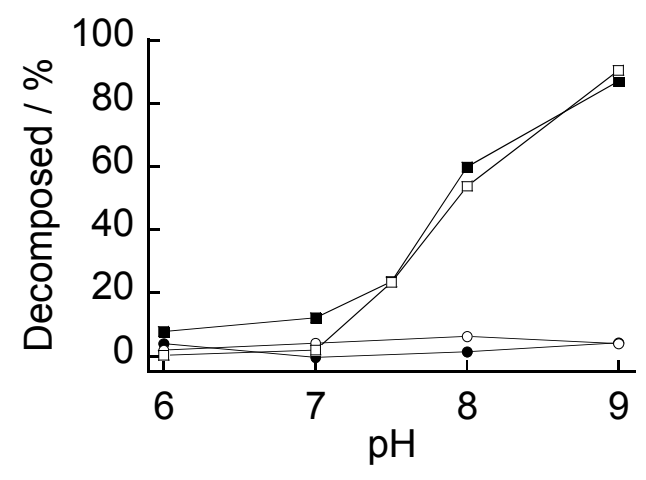

Figure 6. $\mathrm{pH}$-dependent stability of (CMC/19\%PBA-PAMAM $)_{10}(\square),(\mathrm{CMC} / 39 \% \text { PBA-PAMAM })_{10}$ $(\square),(\text { AGA/19\%PBA-PAMAM) } 10(\bigcirc) \text {, and (AGA/39\%PBA-PAMAM) })_{10}$ films $(\bullet)$. The percentages of decomposition after immersing the films in the buffer solutions for two hours are plotted.

LbL films based on boronate ester bonds are often unstable in neutral and acidic solutions because boronate ester bonds are stable only when the boron atom assumes a negatively charged tetragonal form in basic media. For instance, LbL films consisting of PBA-modified poly(acrylic acid) and mannan 
were stable at $\mathrm{pH} 9.0$ whereas the films decomposed at $\mathrm{pH} 8.0$ or lower [27]. In addition, LbL films composed of 39\%PBA-PAMAM and PVA decomposed at $\mathrm{pH} 7.0$ or lower [19]. In this regard, the present results demonstrate that use of anionic polysaccharides, such as AGA and CMC, is effective for stabilizing PBA-PAMAM LbL films in neutral and weakly acidic media.

In this context, it is noteworthy that polyelectrolyte multilayer films can be stabilized by non-electrostatic interactions, such as hydrogen bonds, in spite of charge imbalance between the components [28-30].

\subsection{Sugar-Induced Decomposition}

Sugar-sensitive LbL films have been widely studied for the development of biosensors and delivery systems [22-24,31-35]. LbL films that decompose in response to sugars but are stable under physiological conditions would be highly useful for biomedical applications. PBA-PAMAM films may be destabilized or decomposed when the films are exposed to sugars as a result of the addition of sugars to PBA-PAMAM. We have evaluated the sugar response of the (AGA/19\%PBA-PAMAM) 10 and (AGA/39\%PBA-PAMAM) ${ }_{10}$ films because these films are stable at physiological $\mathrm{pH}$ (see Figure 6). In fact, we have found that these films decomposed in part in fructose solutions.

Figure 7 shows the decomposition behavior of the (AGA/19\%PBA-PAMAM) 10 and (AGA/ $39 \%$ PBA-PAMAM $)_{10}$ films in glucose and fructose solutions at $\mathrm{pH}$ 7.5. Both the films decomposed upon the addition of 5-30 mM fructose, depending on the concentration of fructose. Two different mechanisms may be involved in the film decomposition. First, boronate ester bonds between PBA-PAMAM and AGA in the films were cleaved by competitive binding of fructose to PBA-PAMAM. It is reasonable to assume that some portions of PBA residues in the films are involved in boronate ester bonds with AGA at pH 7.5 whereas others are free from ester bonds. Second, the binding of fructose to free PBA residues enhanced the amount of negative charges of PBA-PAMAM because the $\mathrm{p} K_{\mathrm{a}}$ of boronate esters is usually more acidic than that of free PBA (see Figure 2). The negatively charged PBA-PAMAM induced destabilization of the films owing to the electrostatic repulsion by AGA. In both mechanisms, the formation of boronate ester bonds between PBA-PAMAM and added fructose is responsible for the film decomposition. Thus, it is quite natural that the (AGA/39\%PBA-PAMAM) 10 film was more sensitive to fructose than the film made of 19\%PBA-PAMAM. On the other hand, the binding affinity of glucose to PBA-PAMAM was too low at $\mathrm{pH} 7.5$ to induce film decomposition. In other words, the (AGA/19\%PBA-PAMAM) 10 and (AGA/39\%PBA-PAMAM) 10 films can be decomposed selectively by the addition of fructose.

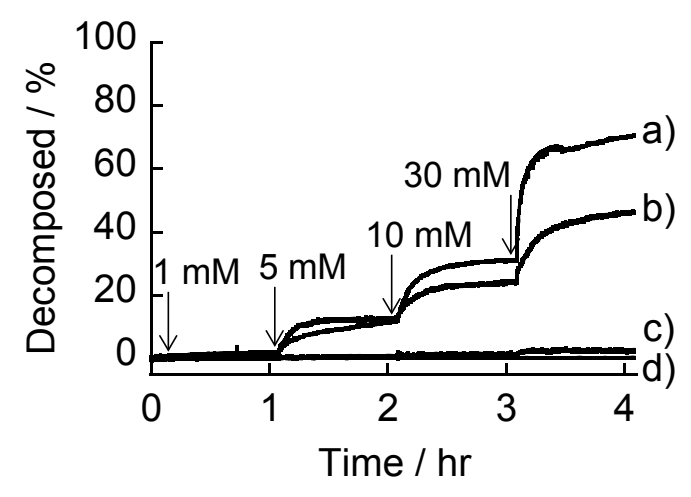

Figure 7. Decomposition of (AGA/19\%PBA-PAMAM) 10 (b,c) and (AGA/39\%PBA-PAMAM) 10 films $(\mathbf{a}, \mathbf{d})$ upon successive addition of fructose $(\mathbf{a}, \mathbf{b})$ and glucose $(\mathbf{c}, \mathbf{d})$ in buffer solutions at $\mathrm{pH}$ 7.5. LbL films were prepared at $\mathrm{pH} 7.5$.

\section{Conclusions}

LbL films comprising PBA-PAMAM and polysaccharides were successfully prepared by the alternate deposition of PBA-PAMAM and AGA or CMC on the surface of a quartz slide. The LbL films were stabilized mainly by the electrostatic affinity between PBA-PAMAM and polysaccharides at 
$\mathrm{pH}$ 6.0-7.0 while boronate ester bonds were involved in the AGA/PBA-PAMAM films at $\mathrm{pH}$ 8.0-9.0. Thus, the AGA/PBA-PAMAM films were stable in the range of $\mathrm{pH}$ 6.0-9.0. The AGA/PBA-PAMAM films decomposed in the presence of 5-30 mM fructose at $\mathrm{pH} 7.5$, whereas glucose did not induce decomposition of the films. The results showed the usefulness of AGA as a counter-polymer in constructing sugar-sensitive PBA-PAMAM films that are stable at physiological $\mathrm{pH}$.

Supplementary Materials: The following are available online at www.mdpi.com/1996-1944/9/6/425/s1.

Author Contributions: Jun-ichi Anzai and Kentaro Yoshida conceived and designed the experiments. Keisuke Suwa performed the experiments. Jun-ichi Anzai prepared manuscript of the paper.

Conflicts of Interest: The authors declare no conflict of interest.

\section{References}

1. Lin, X.; Yang, M.; Jeong, H.; Chang, M.; Hong, J. Durable superhydrophilic coatings formed for anti-biofouling and oil-water separation. J. Membr. Sci. 2016, 506, 22-30. [CrossRef]

2. Huang, J.; Yang, Y.; Shi, H.; Song, Z.; Zhao, Z.; Anzai, J.; Chen, Q. Multi-walled carbon nanotubes-based glucose biosensor prepared by a layer-by-layer technique. Mater. Sci. Eng. C 2006, 26, 113-117. [CrossRef]

3. Zhang, Z.; Liu, J.; Qi, Z.; Lu, D. In situ study of self-assembled nanocomposite films by spectral SPR sensor. Mater. Sci. Eng. C 2015, 51, 242-247. [CrossRef] [PubMed]

4. Yoshida, K.; Sato, K.; Anzai, J. Layer-by-layer polyelectrolyte films containing insulin for pH-triggered release. J. Mater. Chem. 2010, 20, 1546-1552. [CrossRef]

5. Sato, K.; Takahashi, S.; Anzai, J. Layer-by-layer thin films and microcapsules for biosensors and controlled release. Anal. Sci. 2012, 28, 929-938. [CrossRef] [PubMed]

6. Sato, K.; Anzai, J. Dendrimers in layer-by-layer assemblies: Synthesis and applications. Molecules 2013, 18, 8440-8460. [CrossRef] [PubMed]

7. Volodkin, D.; von Klitzing, R.; Moehwald, H. Polyelectrolyte multilayers: Toward cell studies. Polymers 2014, 6, 1502-1527. [CrossRef]

8. Tomita, S.; Sato, K.; Anzai, J. Layer-by-layer assembled thin films composed of carboxyl-terminated poly(amidoamine) dendrimer as a pH-sensitive nano-device. J. Colloid Interface Sci. 2008, 326, 35-40. [CrossRef] [PubMed]

9. Miao, X.; Liu, Y.; Gao, W.; Hu, N. Layer-by-layer assembly of collagen and electroactive myoglobin. Bioelectrochemistry 2010, 79, 187-192. [CrossRef] [PubMed]

10. Inoue, H.; Anzai, J. Stimuli-sensitive thin films prepared by a layer-by-layer deposition of 2-iminobiotinlabeled poly(ethyleneimine) and avidin. Langmuir 2005, 18, 8354-8359. [CrossRef] [PubMed]

11. Anzai, J.; Kobayashi, Y.; Suzuki, Y.; Takeshita, H.; Chen, Q.; Osa, T.; Hoshi, T.; Du, X. Enzyme sensors prepared by layer-by-layer deposition of enzymes on a platinum electrode through avidin-biotin interaction. Sens. Actuators B 1998, 52, 3-9. [CrossRef]

12. Crouzier, T.; Boudou, T.; Picart, C. Polysaccharide-based polyelectrolyte multilayers. Curr. Opin. Colloid Interface Sci. 2010, 15, 417-426. [CrossRef]

13. Suzuki, I.; Egawa, Y.; Mizukawa, Y.; Hoshi, T.; Anzai, J. Construction of positively charged layered assemblies assisted by cyclodextrin complexation. Chem. Commun. 2002, 164-165. [CrossRef]

14. Silva, J.M.; Caridale, S.G.; Costa, R.R.; Alves, N.M.; Groth, T.; Picart, C.; Reis, R.L.; Mano, J.F. pH Responsiveness of multilayered films and membranes made of polysaccharides. Langmuir 2015, 31, 11318-11328. [CrossRef] [PubMed]

15. Aytar, B.S.; Prausnitz, M.R.; Lynn, D.M. Rapid release of plasmid DNA from surfaces coated with polyelectrolyte multilayers promoted by the application of electrochemical potentials. ACS Appl. Mater. Interfaces 2012, 4, 2726-2734. [CrossRef] [PubMed]

16. Sato, K.; Suzuki, I.; Anzai, J. Preparation of polyelectrolyte-layered assemblies containing cyclodextrin and their binding properties. Langmuir 2003, 19, 7406-7412. [CrossRef]

17. Rani, A.; Oh, K.A.; Koo, H.; Lee, H.J.; Park, M. Multilayer films of cationic graphene-polyelectrolytes and anionic graphene-polyelectrolytes fabricated using layer-by-layer self-assembly. Appl. Surf. Sci. 2011, 257, 4982-4989. [CrossRef] 
18. Watahiki, R.; Sato, K.; Suwa, K.; Niina, S.; Egawa, Y.; Seki, T.; Anzai, J. Multilayer films composed of phenylboronic acid-modified dendrimers sensitive to glucose under physiological conditions. J. Mater. Chem. B 2014, 2, 5809-5817. [CrossRef]

19. Suwa, K.; Nagasaka, M.; Niina, S.; Egawa, Y.; Seki, T.; Anzai, J. Sugar response of layer-by-layer films composed of poly(vinyl alcohol) and poly(amidoamine) dendrimer bearing 4-aminophenylboronic acid. Colloid Polym. Sci. 2015, 293, 1043-1048. [CrossRef]

20. Suwa, K.; Sato, K.; Anzai, J. Preparation of multilayer films consisting of glucose oxidase and poly(aminoamine) dendrimer and their stability. Colloid Polym. Sci. 2015, 293, 2713-2718. [CrossRef]

21. Springsteen, G.; Wang, B. A detailed examination of boronic acid-Diol complexation. Tetrahedron 2002, 58, 5291-5300. [CrossRef]

22. Ding, Z.; Guan, Y.; Zhang, Y.; Zhu, X.X. Layer-by-layer multilayer films linked with reversible boronate ester bonds with glucose-sensitivity under physiological conditions. Soft Matter 2009, 5, 2302-2309. [CrossRef]

23. Wang, B.; Liu, Z.; Xu, Y.; Li, Y.; An, T.; Su, Z.; Peng, B.; Lin, Y.; Wang, Q. Construction of glycoprotein multilayers using the layer-by-layer assembly technique. J. Mater. Chem. 2012, 22, 17954-17960. [CrossRef]

24. Zhang, X.; Guan, Y.; Zhang, Y. Dynamically bonded layer-by-layer films for self-regulated insulin release. J. Mater. Chem. 2012, 22, 16299-16305. [CrossRef]

25. Scott, R.W.; Wilson, O.M.; Crooks, R.M. Synthesis, characterization, and applications of dendrimerencapsulated nanoparticles. J. Phys. Chem. B 2005, 109, 692-704. [CrossRef] [PubMed]

26. Maureira, A.; Rivas, B.L. Metal ions recovery with alginic acid coupled to ultrafiltration membrane. Eur. Polym. J. 2009, 45, 573-581. [CrossRef]

27. Levy, T.; Déjugnat, C.; Sukhorukov, G.B. Polymer microcapsules with carbohydrate-sensitive properties. Adv. Funct. Mater. 2008, 18, 1586-1594. [CrossRef]

28. Kharlampieva, E.; Sukhishvili, S.A. Polyelectrolyte multilayers of weak polyacid and cationic copolymer: Competition of hydrogen-bonding and electrostatic interactions. Macromolecules 2003, 36, 9950-9956. [CrossRef]

29. Kharlampieva, E.; Izumrudov, V.A.; Sukhishvili, S.A. Electrostatic layer-by-layer self-assembly of poly(carboxybetaine)s: Role of zwitterions in film growth. Macromolecules 2007, 40, 3663-3668. [CrossRef]

30. Westwood, M.; Noel, T.R.; Parker, R. Environmental responsiveness of polygalacturonic acid-based multilayers to variation of $\mathrm{pH}$. Biomacromolecules 2011, 12, 359-369. [CrossRef] [PubMed]

31. Ma, Y.; Qian, L.; Huang, H.; Yang, X. Buildup of gold nanoparticle multilayer thin films based on the covalent-bonding interaction between boronic acids and polyols. J. Colloid Interface Sci. 2006, 295, 583-588. [CrossRef] [PubMed]

32. De Geest, B.G.; Jones, A.M.; Demeester, J.; De Smedt, S.C. Glucose-responsive polyelectrolyte capsules. Langmuir 2006, 22, 5070-5074. [CrossRef] [PubMed]

33. Manna, U.; Patil, S. Glucose-triggered drug delivery from boronate mediated layer-by-layer self-assembly. ACS Appl. Mater. Interfaces 2010, 2, 1521-1527. [CrossRef] [PubMed]

34. Cambre, J.N.; Sumerlin, B.S. Biomedical applications of boronic acid polymers. Polymer 2011, 52, 4631-4643. [CrossRef]

35. Qi, W.; Yan, X.; Fei, J.; Wang, A.; Cui, Y.; Li, J. Triggered release of insulin from glucose-sensitive enzyme multilayer shells. Biomaterials 2009, 30, 2799-2806. [CrossRef] [PubMed]

(C) 2016 by the authors; licensee MDPI, Basel, Switzerland. This article is an open access article distributed under the terms and conditions of the Creative Commons Attribution (CC-BY) license (http://creativecommons.org/licenses/by/4.0/). 